\title{
Analytical Calculation of the Torque Produced by Transverse Flux Machines
}

\author{
J. R. Anglada ${ }^{1, *}$ and S. M. Sharkh ${ }^{1}$ \\ ${ }^{1}$ Mechatronics Research Group, Engineering Sciences, Faculty of Engineering and the Environ- \\ ment, University of Southampton, Southampton, U.K. \\ *j.renedo-anglada@soton.ac.uk
}

\begin{abstract}
An analytical technique for the calculation of torque produced by a transverse flux machine is developed in this paper. In this technique, the magnets are replaced by their equivalent current sheets and the torque is calculated using the Lorentz force equation. The flux density distribution is calculated using a conformal mapping method that takes into account curvature and slotting. The result is a relatively simple equation that relates torque to the electric and magnetic loadings of the machine and a flux factor that depends on key machine geometrical parameters. The results of the analytical model are verified using finite element analysis and experimental data.
\end{abstract}

\section{Introduction}

The main feature that makes transverse flux machines (TFMs) attractive is their high torque density $[1,2]$. This property stimulated interest in using these machines, which are sometimes referred to as variable reluctance permanent magnet (VRPM) machines [3], for electric vehicle (EV) and ship propulsion [3, 4, 5, 6, 7, 8]. But because of their complicated geometry and low power factor [9] they have not been widely adopted.

However, recent rapid development of renewable energy devices such as wind and marine energy harvesting devices has rekindled interest in using TFMs in these applications. Wind turbines typically have a speed of 5-25 rpm, which makes it necessary to install a gearbox in the drive train to enable the use of traditional electrical generators [10,11, 12]. Marine energy harvesting devices require similarly complicated electrical power take off systems $[13,14]$. In both wind and the marine energy harvesting devices direct drive high torque density generators such as the transverse flux machines would present several advantages in terms of reliability, maintenance costs, longevity and efficiency $[15,16,17]$.

Transverse flux machines are intrinsically three-dimensional and more difficult to model than traditional machines. For this reason most of the modelling and design optimisation studies reported in the literature focus on using numerical techniques including finite elements analysis (FEA) [18]. However, numerical methods do not readily provide the same level of insight that can be gained from analytical models, which remain very useful techniques for initial design optimisation. The insight gained from such analytical model can be key to unlocking the potential of transverse flux machines by enabling their design to be optimised to reduce their complexity and achieve a happy compromise between torque density and power factor $[5,7,9,19]$.

This paper generalizes the torque equation proposed by Harris et al. $[3,4,5,6,7]$ that is based on the fact that the magnets can be represented with current loops [20,21], which allows the 
calculation of torque by applying the Lorentz force $B i L$ equation, where $i$ is the equivalent current of the magnet and $B$ the magnetic field produced by the stator winding. A similar approach has been recently applied for the analysis of axial flux machines [22], in which the BiL principle is applied to the surface current in the modified slotless domain.

Harris et al. assume that the magnets are represented in a 2-dimensional model by single point currents placed at either side of each magnet, at the inner bore of the rotor core-back. The result is a simple equation relating the average torque produced by the machine -assuming square-wave current- to the machine's effective active length $L$, the magnet's mmf $F_{m}$, the armature's mmf $F$ and a flux factor $K_{B}$, which relates the geometrical dimensions of the magnets and stator core to the amount of armature flux contributing to torque production:

$$
T=q\left(\frac{\mu_{0} F}{g}\right) K_{B}\left(2 F_{m}\right) 2 L n\left(\frac{D}{2}\right)=2 q K_{B} B_{s} F_{m} L n D .
$$

where $n$ is the number of pole pairs, $q$ the number of phases, $g$ the effective air-gap length and $D$ the effective diameter. $B_{s}$ is simply expressed as:

$$
B_{s}=\frac{\mu_{0} F}{g} .
$$

The flux factor $K_{B}$, which depends only on the geometric parameters of the air-gap and slots, was estimated by Harris et al. based on analytical calculations of harmonics in slotted developed machines [23] and 2-dimensional FEA. However, the method described in [3] neglects the distributed nature of the magnet's equivalent currents. This, together with the neglect of the effect of curvature can introduce significant errors when calculating $K_{B}$ and torque for machines with relatively large gap to diameter ratios.

This paper uses a complex permeance (CP) method [24, 25, 26, 27] adapted for the calculation of a homopolar field distribution in a TFM and taking into account multiple slot interaction and curvature [28]. The method uses the Schwarz-Christoffel (SC) transformation to obtain an expression for the flux factor $K_{B}$.

Furthermore, the paper derives a generalised expression for $K_{B}$ and hence torque that is more accurate than that developed by Harris et al. [3]. The equivalent currents of the magnets are assumed to be distributed as sheets, rather than single points; the current waveform can take any shape including square and sinusoidal waveforms. The resulting equation can be used to estimate the average torque for any armature current phase advance angle. Therefore, the proposed methodology can be used for the design optimisation of TFMs as shown in [19].

The torque equation proposed in this paper is derived for a particular TFM. However, the principle is completely general and therefore, it can be applied to any kind of PM machine such as permanent magnet actuators or surface PM synchronous machines.

The paper starts with a description of the transverse flux machine developed at the University of Southampton [3, 4, 5, 6, 7], which is used as the particular case study in this paper. Section 3 presents a complex permeance method for the calculation of the homopolar magnetic field distribution in the air-gap produced by the stator windings. This is followed by a derivation of the the torque equation and an expression for the flux factor. Finally, the derived torque equation is validated using FEA and experimental results obtained for the machine under study. 


\section{Description of the Machine}

The magnetic topology of this machine is similar to that of claw-pole machines $[3,4,1,2]$. Fig. 1 shows the radial and axial cross-sections of the machine built at the University of Southampton [3, $4,6,5,7,19]$. The stator has two phases comprising a circular coil each, linking and magnetising 20 laminated C-cores, which modulate the armature's magnetic field to produce a fundamental heteropolar (40 poles) harmonic in the radial direction. The number and width of the C-cores has been carefully selected to maximise the flux utilisation factor and the torque produced by the machine; the torque equation derived in this paper can be used to determine these optimum values of these parameters [7].

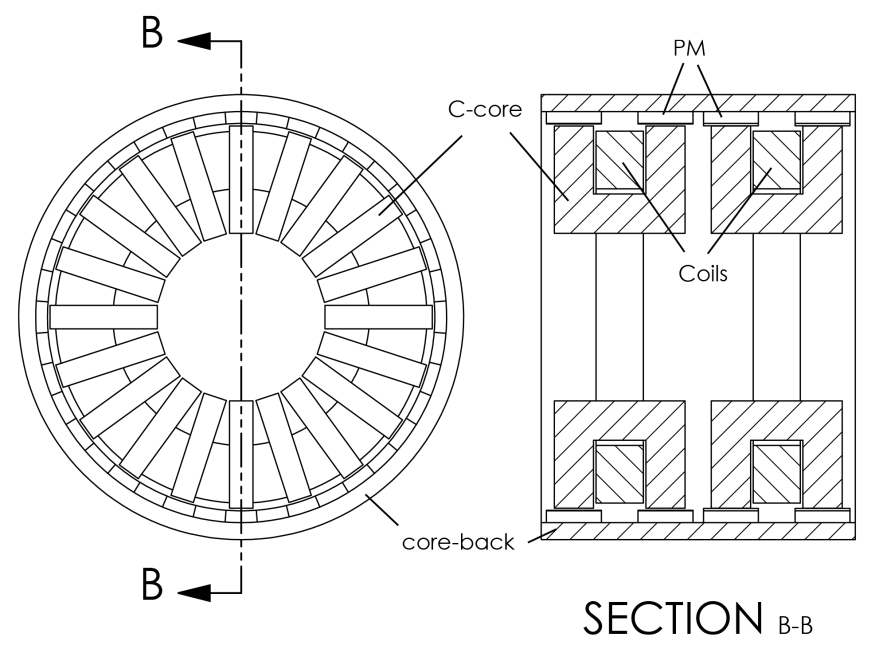

Fig. 1. Front view and cut of a single sided TFM.

The outer rotor comprises a cylindrical steel yoke with 4 arrays of 40 heteropolar magnets each, glued to the inside surface. Each phase is associated with two arrays of magnets: one array positioned over the left-hand $\mathrm{C}$-core legs and the other array, which is spatially anti-phase with the first, is positioned over the right-hand C-Core legs. The two sets of magnet arrays corresponding to the two phases are spatial shifted by 90 electrical degrees (alternatively, the two sets of C-cores could be spatially shifted by 90 electrical degrees). The radial heteropolar flux harmonic interact with the magnets to produce useful torque. The aligned position is defined as the position of the rotor in which the flux passing through the $\mathrm{C}$-cores is maximum.

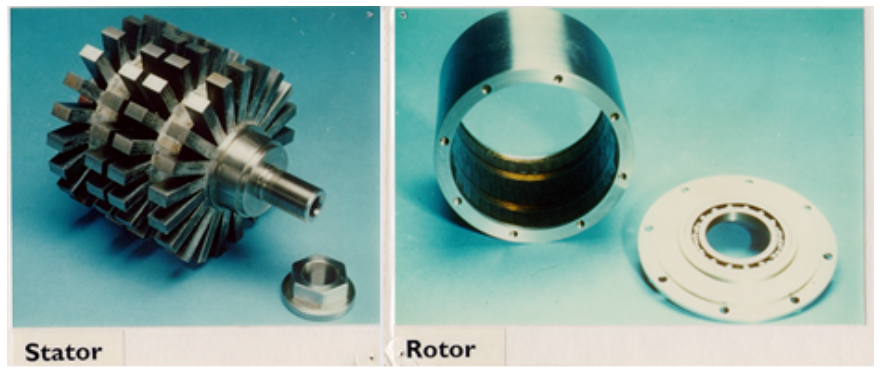

Fig. 2. Picture of the stator and the rotor of the TFM under study.

Fig. 2 shows a picture of the prototype machine. The key geometrical parameters of this device 
are shown in table 1.

Table 1 Parameters of the TFM

\begin{tabular}{lcc}
\hline Quantity & Symbol & Value \\
\hline Stator radius & $R_{s}$ & $73 \mathrm{~mm}$ \\
Rotor radius & $R_{r}$ & $78.5 \mathrm{~mm}$ \\
Clearance gap & $c_{g}$ & $1 \mathrm{~mm}$ \\
Magnet thickness & $d_{m}$ & $4.5 \mathrm{~mm}$ \\
Effective air-gap & $g_{z}$ & $5.5 \mathrm{~mm}$ \\
Pole pitch & $\theta_{\lambda}$ & $18^{\circ}$ \\
Tooth pitch & $\theta_{t}$ & $7.02^{\circ}$ \\
Slot pitch & $\theta_{s}$ & $10.98^{\circ}$ \\
Number of C-cores & $n$ & 20 \\
Number of turns & $N_{t}$ & 230 \\
Number of phases & $q$ & 2 \\
\hline
\end{tabular}

The effective air-gap takes into account that the permeability of the magnets is almost the same as the permeability of vacuum. Therefore, the effective magnetic air-gap, as shown in Fig. 3, is calculated as:

$$
g_{z}=d_{m}+c_{g}
$$

There are many different topologies of TFMs and some of them present more complicated flux-concentrating topologies. However, this machine was chosen as a case study because its configuration is relatively simple and therefore it can be used to illustrate the calculation of torque using the proposed methodology.

\section{Analytical Calculation of the Magnetic Field in the Air-Gap}

In this paper the magnetic field distribution in the air-gap is calculated using the complex permeance (CP) method [24, 25, 26]. The calculation of the CP function for TFMs is shown in [28] and the same notation is used in this paper.

In our machine we need to find the function $\lambda(\theta, r)$ that modulates the scalar value of $B_{s}(t)$ as defined by Harris et al.:

$$
B_{s}(t)=\frac{\mu_{e q} \mu_{0} F(t)}{g_{z}}
$$

where $F(t)$ is the instantaneous magneto-motive force (MMF) produced by the stator windings and $g_{z}$ is the air-gap length and $\mu_{e q}$ is the equivalent permeability, which depends on the relative permeability of the PMs $\left(\mu_{r}\right)$ and the magnet thickness to effective air-gap ratio. It is calculated as follows

$$
\mu_{e q}=\frac{g_{z}}{\frac{d_{m}}{\mu_{r}}+c_{g}}
$$

The function $\lambda(\theta, r)$ can be formulated in such a way that the real part corresponds to the radial component and the imaginary to the tangential one. In this paper the variable $\theta$ is expressed in 
electrical radians. Accordingly, the expression of the magnetic field distribution in the air-gap of the $z$-plane expressed as a vector is

$$
\vec{B}(t, \theta, r)=B_{s}(t)\left[\operatorname{Re}\{\lambda(\theta, r)\} \vec{u}_{r}+\operatorname{Im}\{\lambda(\theta, r)\} \vec{u}_{\theta}\right] .
$$

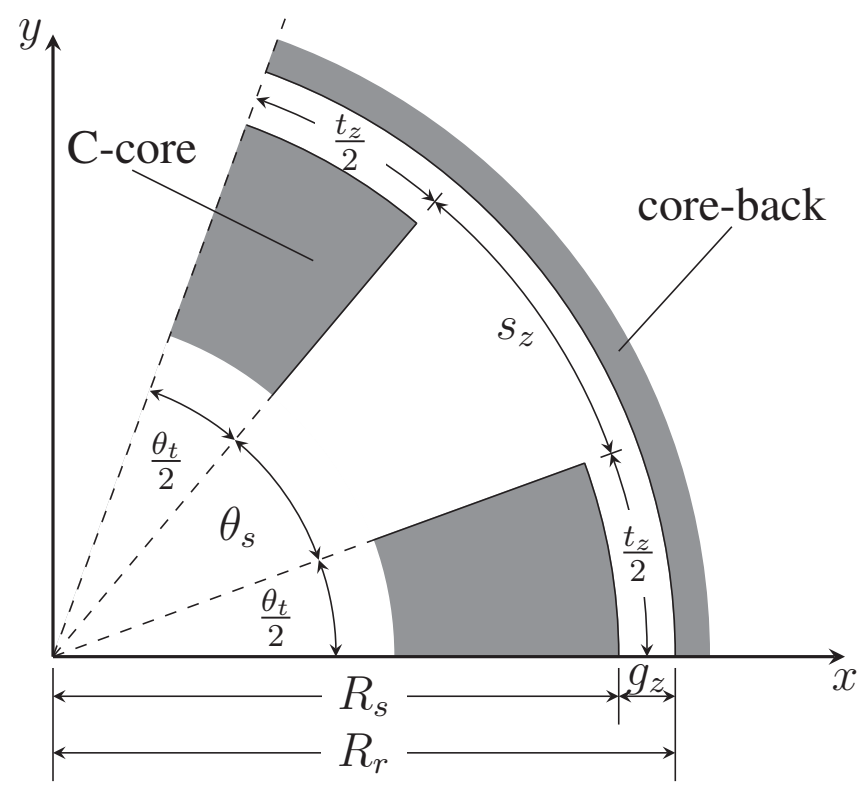

Fig. 3. Geometry of the TFM considered for the CP function, z-plane.

This method is based on the assumption of infinitely permeable iron core, therefore the effect of saturation is not considered in this paper. For the effect of slotting we are assuming very deep slots with radial teeth, as shown in Fig. 3, when in reality the C-cores' sides are parallel.

The proportional-logarithmic transformation maps the cylindrical domain into a rectangular one and its permeance function, $\lambda_{\log }$, can be simply expressed as:

$$
\lambda_{\log }(r)=\frac{R_{g}}{r} .
$$

On the other hand, there is not an explicit expression for the Schwarz-Christoffel transformation. However, because of the symmetry of the boundary conditions the permeance function, $\lambda_{S C}$, can be expressed as:

$$
\lambda_{S C}(\theta, r)=\lambda_{r}(\theta, r)+j \lambda_{\theta}(\theta, r)
$$

where:

$$
\begin{gathered}
\lambda_{r}(\theta, r)=\bar{\lambda}_{r}\left[1+\sum_{n=1}^{\infty} \gamma_{n}(r) \cos (n \theta)\right] \\
\lambda_{\theta}(\theta, r)=\sum_{n=1}^{\infty} \lambda_{\theta n}(r) \sin (n \theta)
\end{gathered}
$$


Therefore, the expression of the radial component of the magnetic field distribution of the airgap is given by

$$
B_{r}(t, \theta, r)=B_{s}(t) \bar{\lambda}_{r}\left[1+\sum_{n=1}^{\infty} \gamma_{n}(r) \cos (n \theta)\right] \lambda_{\log }(r)
$$

By evaluating $\lambda_{S C}(\theta, r)$ we can calculate the coefficients that determine the CP function (11) as shown in [28].

\section{Torque Equation}

Fig. 4 shows a developed model of the TFM with dots and crosses representing the equivalent currents placed along the edges (equivalent currents are shown for one magnet only).

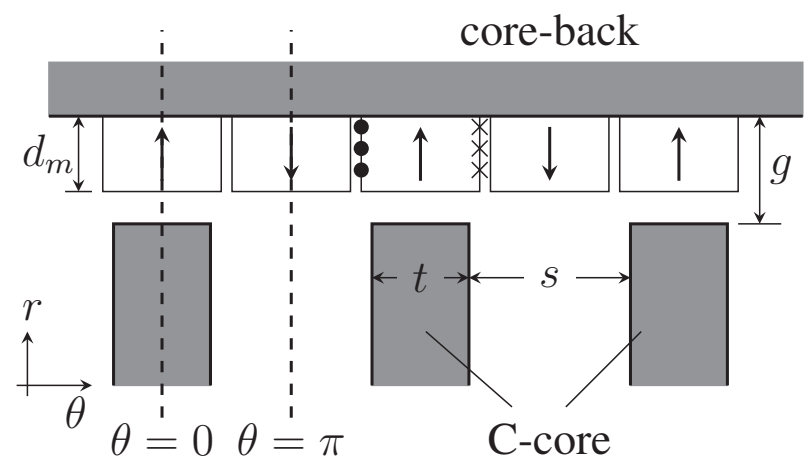

Fig. 4. Developed model of the TFM.

The basic approach is to firstly calculate the average torque of a single current loop at an arbitrary distance from the core-back (represented by $r_{\delta}$ ) and then calculate the total torque by integrating the resulting expression over the length of the magnets' equivalent current sheets. Radially magnetised permanent magnets can be replaced by equivalent currents for the calculation of the magnetic field distribution in the air-gap, as shown by Boules [20]. The linear current density $J$ equal to the magnetisation of the material $\mathcal{M}$ as described in [20,21].

\subsection{Torque Produced by a Current Loop}

If we consider a current loop that represents a layer of the magnet and one C-core head, this is the same as saying that we will calculate the force along the arc $r=r_{\delta}$ as shown in Fig. 5. The average tangential force of half of a fundamental electrical period can be calculated using the $B i L$ principle; it is necessary to integrate only over half a period because at that point the sign of the current will change and due to the symmetry the same torque will be produced in the second half of the period. The radial magnetic field $B_{r}(t, \theta, r)$ is produced by the current in the winding, $L$ is the active $\mathrm{C}$-core length and $i_{\delta}$ is the equivalent current of the magnets at $r_{\delta}{ }^{1}$.

The tangential force has two components: the positive force of the currents going out of the paper (dots) and the negative force that comes from the opposite currents (crosses) as it is shown

\footnotetext{
${ }^{1}$ The $\operatorname{arc} r_{\delta}$ represents any arc in the air-gap, the total torque is calculated in the following section by integrating the differential torque along the magnets' thickness.
} 
in Fig. 5. Because the positive currents, $i_{\delta}$, (dots in the figure) are located under the teeth and the negative currents, $-i_{\delta}$, (crosses) are located in the slot regions the total force is positive because the magnetic field is stronger under a tooth than that in the slot regions.

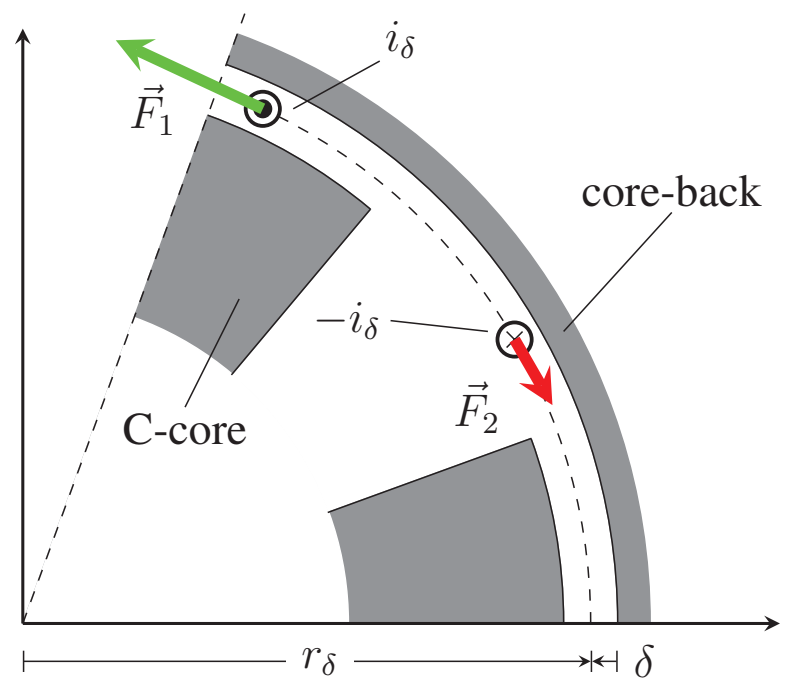

Fig. 5. Forces applied to the equivalent current loop at a distance $\delta$.

The average force experienced by $i_{\delta}$ and $-i_{\delta}$ in Fig. 5 over half of an electrical cycle can be calculated as

$$
F_{\delta}=\frac{1}{\pi}\left\{\int_{-\frac{\pi}{2}}^{\frac{\pi}{2}} B_{r}\left(t, \theta, r_{\delta}\right) i_{\delta} L d \theta-\int_{\frac{\pi}{2}}^{\frac{3 \pi}{2}} B_{r}\left(t, \theta, r_{\delta}\right) i_{\delta} L d \theta\right\} .
$$

We can define $B_{s c}(\delta)$ as the magnetic field of the slotless geometry corrected for the curvature at a distance $\delta$ according to (7)

$$
B_{s c}(\delta)=\frac{\mu_{0} F}{g} \frac{R_{g}}{r_{\delta}}=B_{s} \frac{R_{g}}{r_{\delta}} .
$$

Using $B_{s c}(\delta)$ and considering that $\phi$ is the phase advance angle of the current (angle between the emf $E$ and $I$ ) we can express the force as follows

$$
F_{\delta}=i_{\delta} L B_{s c}(\delta) \cos (\phi) K_{B}(\delta),
$$

where $K_{B}(\delta)$ is called the flux factor for the loop at at distance $\delta$ and it only depends on the geometry. From (12), (13) and (14) the general expression of the flux factor is as follows

$$
K_{B}(\delta)=\frac{1}{B_{s}(\delta) \pi \cos (\phi)} \int_{-\frac{\pi}{2}}^{\frac{\pi}{2}}\left\{B_{r}\left(t, \theta, r_{\delta}\right)-B_{r}\left(t, \theta+\pi, r_{\delta}\right)\right\} d \theta .
$$

Each equivalent current loop is moving along an arc $r=r_{\delta}$. Therefore, the average torque over half a period is simply the product of the average force multiplied by the radius:

$$
T_{\delta}=F_{\delta} r_{\delta}
$$


Considering that $n$ is the number of C-cores (also the number of pole pairs) and that each C-core has two heads that link with two rows of magnets, the total torque per phase can be expressed as

$$
T_{\delta}=n L D_{g} B_{s} i_{\delta} K_{B}(\delta) \cos (\phi)
$$

Assuming that the system is linear, then the total instantaneous torque is simply the superposition of those produced by all the phases. Therefore, the total average torque is simply the multiplication of the number of phases by the average torque of one phase. However, the torque ripple depends significantly on the relative phase angles of harmonic components of the torque produced by the different phases and to obtain the total instantaneous torque is necessary to add them as vectors.

It is important to note that in (17) $n, L, D_{g}$ and $\cos (\phi)$ are known parameters of the machine and do not depend on $r_{\delta}$. Furthermore, the average torque is calculated by integrating Lorentz's equation of force over half a period for each phase independently. Therefore, there are two effects that are not being captured by this approach. Firstly, the cogging torque is neglected. Secondly, the torque ripple which depends on the relative position of the $q$ phases and the harmonic content

of their mmf. While these do not affect the average torque they may be important if vibrations are an issue.

\subsection{Total Torque}

The total average torque produced by one phase can be obtained by integrating (14) along the magnet width, $d_{m}$. If we consider $\delta$ as the distance from the equivalent current loop to the coreback the total torque can be expressed as the following

$$
T=\int_{0}^{d_{m}} d T_{\delta}=\int_{0}^{d_{m}} n L D_{g} B_{s} K_{B}(\delta) \cos (\phi) d i_{\delta} .
$$

The differential current at the edge of the magnet can be expressed in terms of the magnetisation, $\mathcal{M}$, taking into account that there are two adjacent magnets, as

$$
d i_{\delta}=2 \mathcal{M} d \delta .
$$

Therefore the total torque can be expressed as follows

$$
T=2 n L D_{g} B_{s} \mathcal{M} d_{m} \cos (\phi)\left[\frac{1}{d_{m}} \int_{0}^{d_{m}} K_{B}(\delta) d \delta\right],
$$

where $\mathcal{M} d_{m}=F_{m}$ is the total MMF of one permanent magnet and the term inside the brackets can be defined as the total flux factor:

$$
K_{B}^{\text {Total }}=\frac{1}{d_{m}} \int_{0}^{d_{m}} K_{B}(\delta) d \delta
$$

The expression of the average torque produced by a machine with $q$ phases is then

$$
T=2 q K_{B}^{\text {Total }} B_{s} F_{m} L n D_{g} \cos (\phi) .
$$

This expression has the same shape as (1) but with the calculation of the flux factor considers several aspects that were previously neglected, such as the force distributed all through the magnet 
thickness, the effect of curvature and the shape of the MMF waveform. Also, the cosine of the angle between the MMF and the q-axis of the rotor $\cos (\phi), \phi$ is the phase advance angle, appears in (22).

If the magnets are axially longer than the teeth (Fig. 6), then the fringing flux in the axial direction will be actually producing torque. To estimate the equivalent active length of the Ccore in the axial direction we can use the results reported by Markovic et al. [29] in which the permeance of a tooth to tooth structure is calculated. In [29], the conformal transformation is derived and also an approximation is obtained using a fitting formula, which in this case is simply a linear function. Considering a 2D model of a C-core tooth against the core-back as shown in Fig. 6 , then based on Markovic's formula, the permeance per unit length can be approximated as

$$
\Lambda=\mu_{0} \frac{l_{\text {core }}}{g_{z}}\left(1+0.384 \frac{g_{z}}{l_{\text {core }}}\right),
$$

where $l_{\text {core }}$ the $\mathrm{C}$-core head width, Fig. 6 . In the previous deduction of the torque equation it was assumed that the field is constant in the perpendicular direction (two-dimensional approximation). Considering a magnetic potential difference $\Theta$ between the tooth and the core-back the flux per unit length is given by

$$
\Phi_{t}=\Theta \Lambda
$$

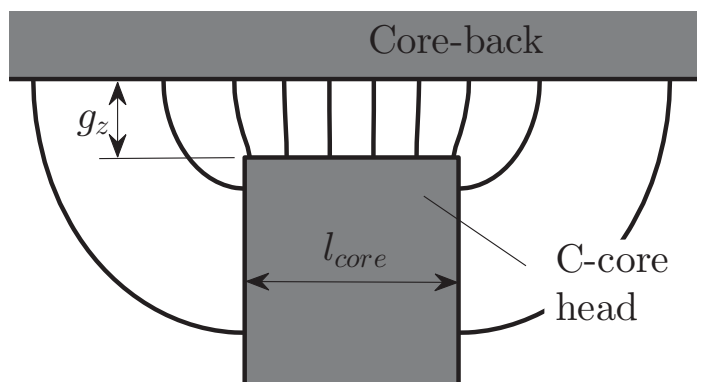

Fig. 6. Fringing around the C-core head, axial geometry considered to obtain the equivalent length.

Therefore we can define an equivalent axial length, $L_{e q}$, as

$$
\Phi_{t}=B_{t} L_{e q}
$$

where $B_{t}=\mu_{0} \Theta / g_{z}$. The flux per unit length is equal to that obtained using the permeance (23). Therefore the equivalent length is given by

$$
L_{e q} \approx l_{\text {core }}\left(1+0.384 \frac{g_{z}}{l_{\text {core }}}\right) \text {. }
$$

This equivalent length takes into account that the fringing flux is actually producing torque when the magnets are longer than the $\mathrm{C}$-core head, $l_{\text {core }}$, and therefore this value should be used in (21). However, when the magnet length, $L_{\text {mag }}$, is not longer than $l_{\text {core }}$ not all the fringing flux is effectively producing torque; in this case the equivalent length should be the magnet's axial length as a first approximation. 


\section{Flux Factor}

The flux-factor is the term in (22) that has all the information about the electro-mechanical interaction that takes place in the air-gap and determines how much of the total flux is effectively producing torque. This coefficient depends only on the complex permeance function that includes the information about the geometry and the waveform of the MMF. In this paper two different MMF cases are considered: a square wave MMF and a sinusoidal MMF, as shown in Fig. 7.

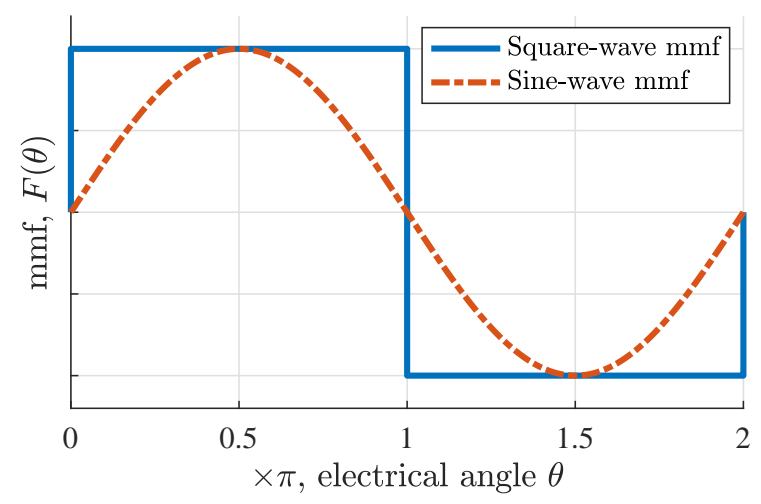

Fig. 7. Shape of the two mmf waveforms, $F(\theta)$, considered in this for the calculation of the flux factor.

\subsection{Flux Factor of a Single Current Loop}

In this section, the flux factor is calculated at an arbitrary distance $\delta$ to the core-back as illustrated in Fig. 5.

Solving the integral in (15) and considering that for a square-wave MMF the expression of the flux-factor is the following:

$$
K_{B}(\delta)=\frac{4}{\pi} \bar{\lambda}_{r}\left[\gamma_{1}(\delta)-\frac{\gamma_{3}(\delta)}{3} \frac{\cos (3 \phi)}{\cos (\phi)}+\frac{\gamma_{5}(\delta)}{5} \frac{\cos (5 \phi)}{\cos (\phi)}-\ldots\right] .
$$

It is important to remember that this is the expression at a particular arc and that to evaluate the integral for the total flux factor $\bar{\lambda}_{r}$ and $\gamma_{n}(\delta)$ vary with $r_{\delta}$.

If the MMF of the winding is a sinusoidal function in synchronism with the rotation of the machine, then the instantaneous magnetic field is expressed as follows:

$$
B_{s}(t)=B_{s o} \cos (\omega t+\phi),
$$

with

$$
B_{s o}=\frac{\mu_{e q} \mu_{0} \hat{F}}{g_{z}}
$$

where $\hat{F}$ is the peak value of the MMF. Accordingly, the magnetic field distribution can be expressed as

$$
B_{r}(\theta, \delta)=B_{s o} \lambda_{\log }(\delta) \cos (\theta+\phi) \bar{\lambda}_{r}\left[1+\sum_{n=1}^{\infty} \gamma_{n}(\delta) \cos (n \theta)\right]
$$


where $\phi$ is the phase advance angle of the MMF. In this situation when we substitute (30) into (15) we obtain the expression of the flux factor for a sine-wave MMF:

$$
K_{B}(\delta)=\bar{\lambda}_{r} \gamma_{1}(\delta)
$$

\subsection{Total Flux Factor}

The total flux factor that takes into account all the magnet thickness is obtained by evaluating the integral in (21). Because the magnetisation of the PM is constant the integration over $d_{m}$ is equivalent to obtaining the average value of the amplitude of the harmonics. Accordingly the expression of the total flux factor for square-wave and sinusoidal waveforms respectively is

$$
\begin{gathered}
K_{B}^{\text {Total }}=\frac{4}{\pi} \bar{\lambda}_{r}\left[\gamma_{1}^{a v}-\frac{\gamma_{3}^{a v}}{3} \frac{\cos (3 \phi)}{\cos (\phi)}+\frac{\gamma_{5}^{a v}}{5} \frac{\cos (5 \phi)}{\cos (\phi)}-\ldots\right] \\
K_{B}^{\text {Total }}=\bar{\lambda}_{r} \gamma_{1}^{a v}
\end{gathered}
$$

where $\gamma_{n}^{a v}$ is the average value of the $n$ harmonic over the magnet thickness calculated as follows:

$$
\gamma_{n}^{a v}=\frac{1}{d_{m}} \int_{0}^{d_{m}} \gamma_{n}(\delta) d \delta
$$

\section{Case Study}

The method described in the previous sections was applied to the geometry of the TFM built at the University of Southampton $[3,4,5,6,7]$. The geometric parameters considered for the calculations are shown in table 1.

The windings of the machine have $N_{t}=230$ turns in the configuration analysed in this paper and the rated current $I$ is $10 \mathrm{~A}$. The MMF across the gap under each $\mathrm{C}$-core head is calculated as follows

$$
F=\frac{1}{2} N_{t} I=1150 \mathrm{~A},
$$

therefore the magnetic field as defined by Harris et al.

$$
B_{s}=\frac{\mu_{e q} \mu_{0} F}{g_{z}} \approx 0.276 \mathrm{~T}
$$

The permanent magnets are made of the neodymium-iron-boron type with $B_{r}=1.05 \mathrm{~T}$ and $\mu_{r} \approx 1.05$. Assuming linearity in the properties of the material [20], the equivalent current of the PMs is calculated as follows:

$$
F_{m}=\mathcal{M} d_{m}=\frac{B_{r}}{\mu_{r} \mu_{0}} d_{m} \approx 3590 \mathrm{~A} .
$$

The active $\mathrm{C}$-core length is calculated as it is described in section 4 considering that the magnet width is $21 \mathrm{~mm}$, which is higher than the C-core head width, $l_{\text {core }}$, that is $15 \mathrm{~mm}$. Therefore, the equivalent length is calculated as follows

$$
L_{e q} \approx l_{\text {core }}+0.384 g_{z} \approx 17.11 \mathrm{~mm}
$$




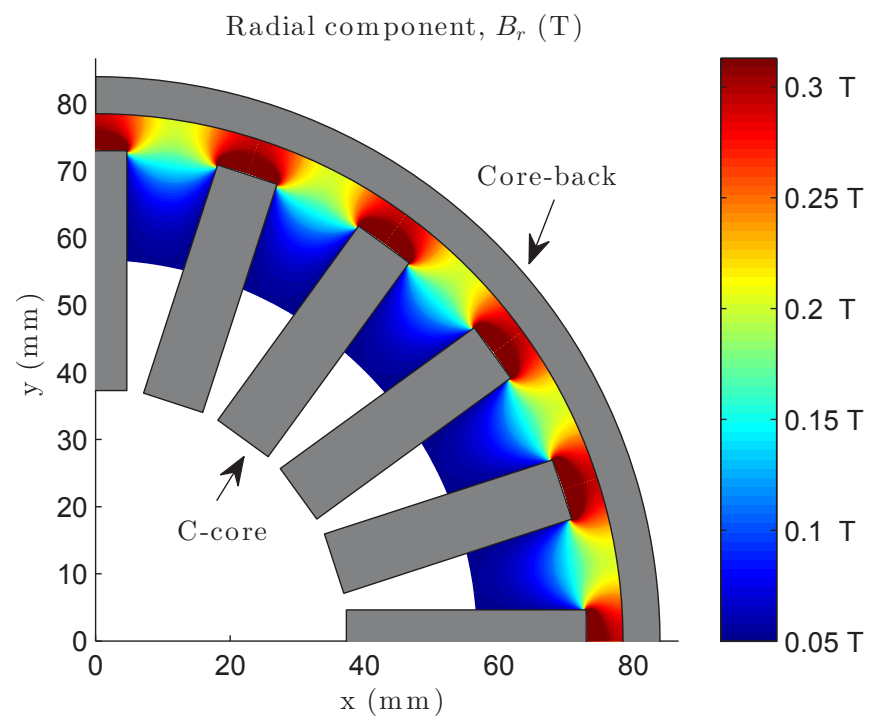

Fig. 8. Radial component of the stator windings magnetic field distribution in the air-gap obtained using the CP function in a quarter of the machine.

This methodology was implemented in MATLAB. Fig. 8 shows the radial component of the stator windings' magnetic field distribution in the air-gap obtained using the CP function.

For the purpose of validation 2D and 3D FEA models were built. The radial and tangential components of the stator windings' magnetic field distribution in the middle of the air-gap, obtained using the $\mathrm{CP}$ function, 3D FEA and 2D FEA; are shown in Fig. 9. It can be appreciated that there is a good agreement between the three methodologies.

One of the limitations of the methodology presented in this paper is that the cogging torque cannot be effectively calculated. The cogging torque obtained using 3D FEA and the experimental data are shown in Fig. 10; it can be appreciated that the cogging torque of one phase is large. However, since the two phases are shifted by 90 electrical degrees the total cogging torque reduces significantly. The total cogging torque obtained using 3D FEA has a similar amplitude to the experimental data but there are some discrepancies between the experimental and FEA waveforms that may be due to the magnetic coupling of the phases or the mechanical tolerance of the prototype.

The static torque of the machine with only phase excited is shown in Fig. 11. The analytical results obtained using the torque equation (solid line) have been corrected by adding the cogging torque from the 3D FEA simulation.

Using the CP function shown in Fig. 8 the value of the flux factor for a square-wave current is

$$
K_{B}^{\text {Total }} \approx 0.3105
$$

The results obtained from the torque equation can be compared with the studies presented in $[3,4]$. The calculation of the flux factor proposed in [3] considers the magnets to be represented by point currents (in the 2D section) at the inner bore of the rotor core-back. Then, the amplitude of the magnetic field harmonics can be obtained from [23] to calculate $K_{B}$ to be 0.275 , which corresponds to the torque equation - Harris line in Fig. 12. However, it is mentioned in [3] that the equivalent current is distributed along the air-gap which the authors from [3] suggested could increase $K_{B}$ to 0.334 without providing details of the calculation. This value is an overestimate compared to the value of $K_{B}^{\text {Total }}$ in (39). 

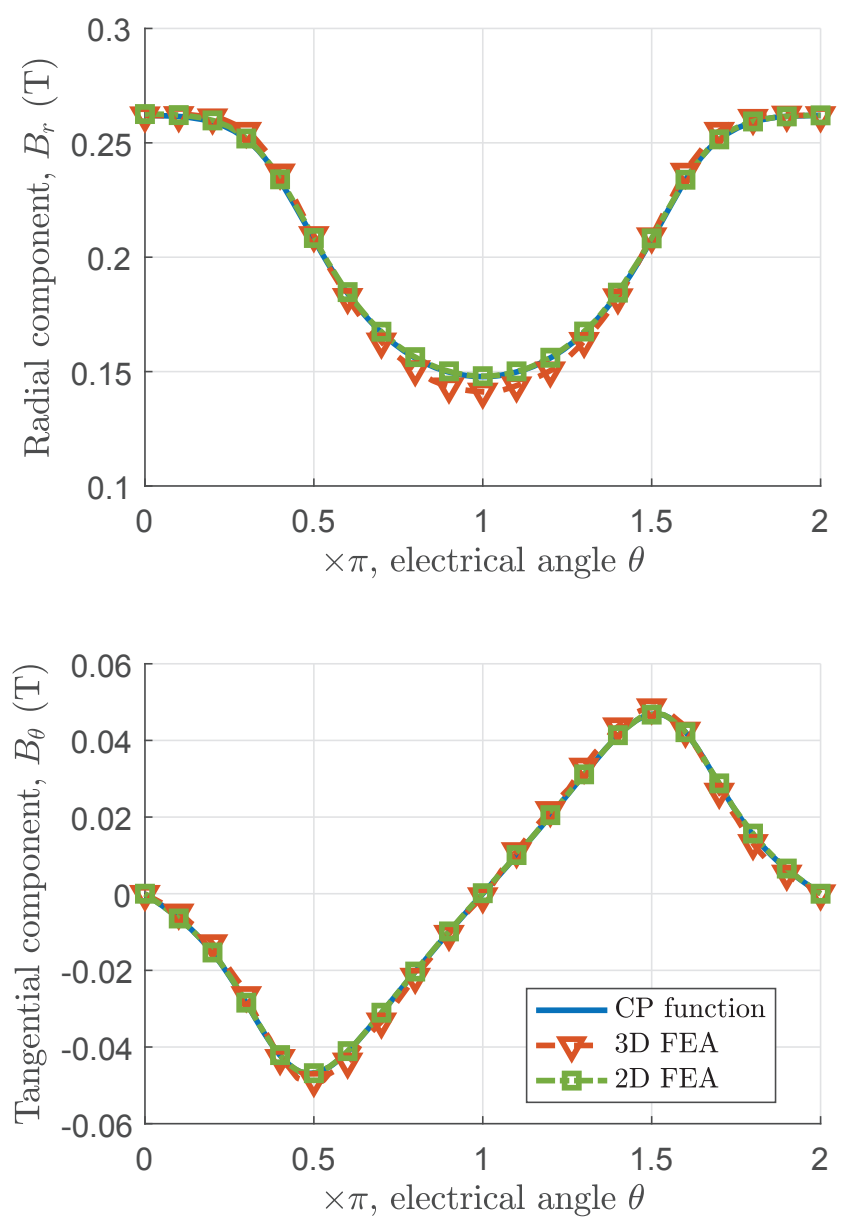

Fig. 9. Radial component, $B_{r}$, and tangential component, $B_{\theta}$, of the stator windings' magnetic field distribution in the middle of the air-gap.

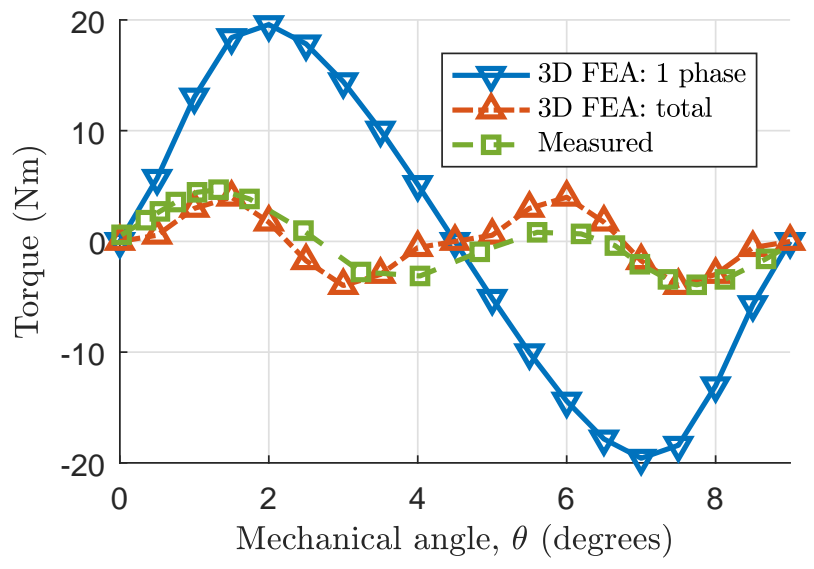

Fig. 10. Cogging torque obtained using 3D FEA (for one phase and for two phases) and experimental results. 


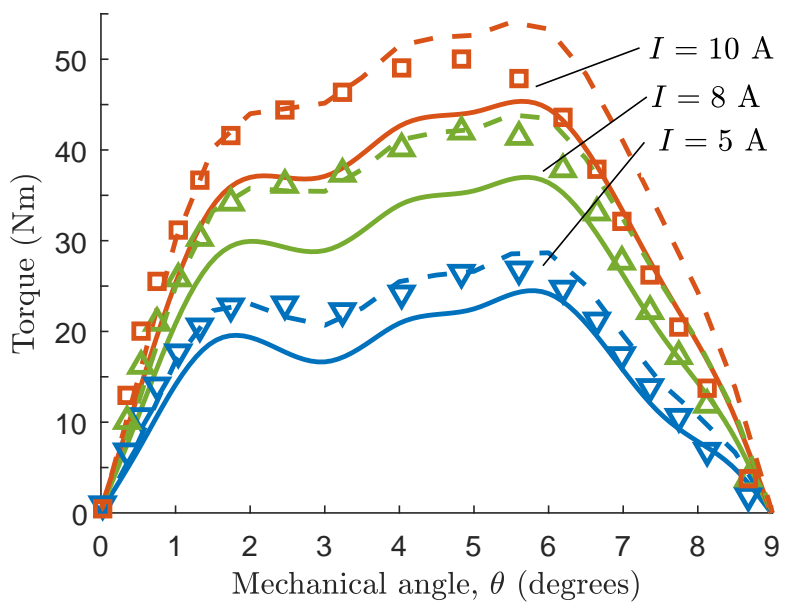

Fig. 11. Static torque calculated using the proposed torque equation (solid line), 3D FEA (dashed line) and experimental data (markers).

Fig. 12 shows the average torque of one phase of the TFM obtained from the generalised torque equation presented in this paper, Harris' torque equation [3], the value measured in the actual device and 3D FEA calculations. The method proposed in this paper is closer to FEA and experimental data in the linear region below saturation (the rated current of this machine is $10 \mathrm{~A}$ ) than that obtained by Harris, which neglects curvature and the spatial distribution of the magnets' equivalent current. The analytical method predicts slightly lower torque values than the 3D FEA and experimental data probably because of the conservative calculation of $L_{e q}$ using (26).

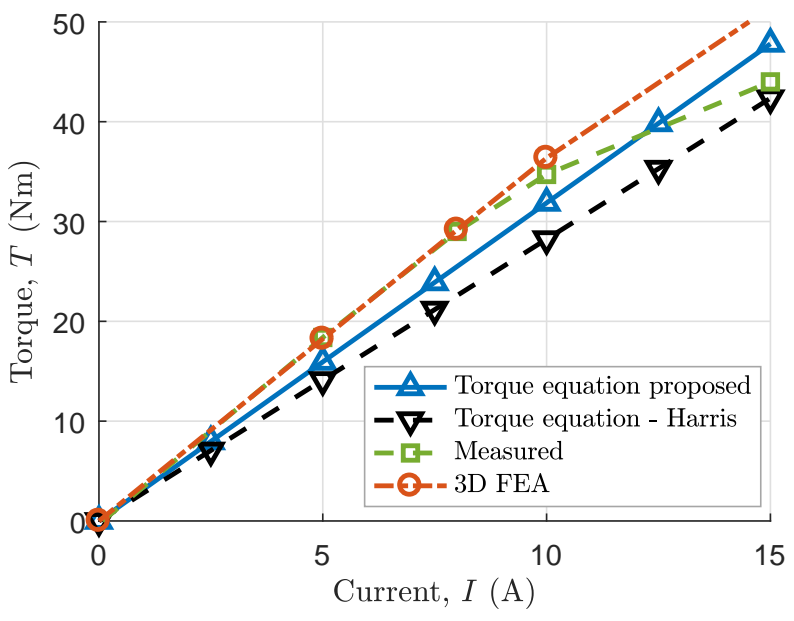

Fig. 12. Average torque of the TFM obtained with the torque equation presented this paper, Harris et al. torque equation, the value measured in the lab and 3D FEA [3, 4].

Fig. 13 shows the real part of the $\mathrm{CP}$ function, $\operatorname{Re}\left\{\lambda_{S C}\right\}$, for $\delta=0 \mathrm{~mm}$ (over the coreback), $\delta=d_{m} / 2$ (middle of the magnet width) and $\delta=d_{m}$ (over the surface of the magnet). It can be appreciated that the amplitude of the harmonics increases as $\delta$ increases. Since the flux factor depends on the amplitude of the harmonics, considering only the top of the core-back 
will underestimate the average torque. Harris et al. [3, 4] mentioned this effect but it was not calculated exactly. The proposed methodology considers this effect accurately when calculating the flux factor.

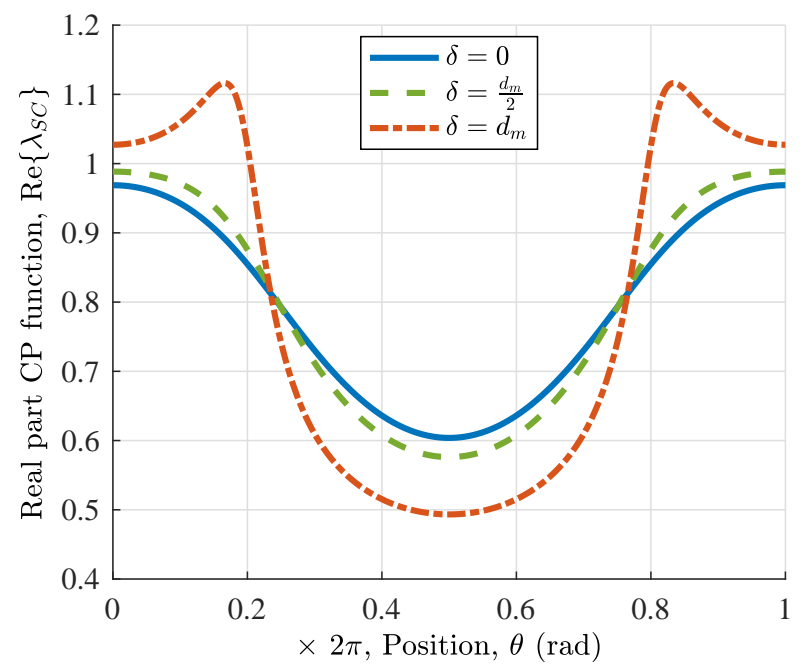

Fig. 13. Real part of the CP function associated to the $S C$ transformation, $\operatorname{Re}\left\{\lambda_{S C}\right\}$, as a function of the position, $\theta$, for three different lengths, $\delta$.

\section{Conclusions}

This paper presents an analytical method for the calculation of torque of PM machines that is particularly useful for transverse flux machines. The method shares the background theory described in $[3,4]$. However, in this paper the PMs are represented as current sheets rather than current points and the calculation of the magnetic field distribution in the air-gap is obtained using a complex permeance function tailored to a homopolar field distribution. These aspects produce more accurate results.

The results of the analytical model presented in this paper are validated by experimental and 3D FEA data. There is good agreement between the proposed analytical method, 3D FEA and the experimental data in the unsaturated region.

\section{Aknowledgement}

This work was supported by EPSRC and by TSL Technology Ltd.

The authors would like to thank Dr. Mike Yuratich and the team of TSL Technology Ltd. for their help during this project.

\section{References}

[1] H. Weh and R. Mayer. Synchronous machine with permanent magnet excitation as a flexible propulsion motor with high efficiency. In Electrical Machines (ICEM), 1984 International Conference on, 1984. 
[2] H. Weh and H. May. Achievable force densities for permanent magnet excited machines in new configurations. In Electrical Machines (ICEM), 1986 International Conference on, 1986.

[3] M. R. Harris and B. C. Mecrow. Variable reluctance permanent magnet motors for high specific output. In Electrical Machines and Drives, 1993. Sixth International Conference on (Conf. Publ. No. 376), pages 437-442, Sep 1993.

[4] M. R. Harris and G. H. Pajooman. Electric motors with heteropolar permanent magnets and homopolar windings: computational study of performance limits. In Electrical Machines and Drives, 1995. Seventh International Conference on (Conf. Publ. No. 412), pages 237241, Sep 1995.

[5] M. R. Harris, G. H. Pajooman, and S. M. A. Sharkh. Comparison of alternative topologies for vrpm (transverse-flux) electrical machines. In New Topologies for Permanent Magnet Machines (Digest No: 1997/090), IEE Colloquium on, pages 2/1-2/7, Jun 1997.

[6] M. R. Harris, G. H. Pajooman, and S. M. Abu Sharkh. Performance and design optimisation of electric motors with heteropolar surface magnets and homopolar windings. IEE Proceedings - Electric Power Applications, 143(6):429-436, Nov 1996.

[7] G. H. Pajooman. Performance assessment and design optimisation of VRPM (transverse flux) machines by finite element computation. PhD Thesis, University of Southampton, 1997.

[8] G. Henneberger and M. Bork. Development of a new transverse flux motor. In New Topologies for Permanent Magnet Machines (Digest No: 1997/090), IEE Colloquium on, pages 1/1-1/6, Jun 1997.

[9] M. R. Harris, H. G. Pajooman, and S.M. Abu Sharkh. The problem of power factor in VRPM (transverse-flux) machines. In Electrical Machines and Drives, 1997 Eighth International Conference on (Conf. Publ. No. 444), number 444, pages 386-390. IET, 1997.

[10] J. Sopanen, V. Ruuskanen, J. Nerg, and J. Pyrhonen. Dynamic torque analysis of a wind turbine drive train including a direct-driven permanent-magnet generator. IEEE Transactions on Industrial Electronics, 58(9):3859-3867, Sept 2011.

[11] H. Polinder, F. F. A. van der Pijl, G. J. de Vilder, and P. J. Tavner. Comparison of direct-drive and geared generator concepts for wind turbines. IEEE Transactions on Energy Conversion, 21(3):725-733, Sept 2006.

[12] E. Spooner, P. Gordon, J. R. Bumby, and C. D. French. Lightweight ironless-stator pm generators for direct-drive wind turbines. IEE Proceedings - Electric Power Applications, 152(1):17-26, Jan 2005.

[13] M. A. Mueller, H. Polinder, and N. Baker. Current and novel electrical generator technology for wave energy converters. In 2007 IEEE International Electric Machines Drives Conference, volume 2, pages 1401-1406, May 2007.

[14] N Hodgins, A Mcdonald, J Shek, O Keysan, and M Mueller. Current and Future Developments of the C-GEN Lightweight Direct Drive Generator for Wave \& Tidal Energy. Proceedings of the 8th European Wave and Tidal Energy Conference, Uppsala, Sweden, pages 352-359, 2009. 
[15] M.A. Mueller and N. Hodgins. Designing the C-GEN lightweight direct drive generator for wave and tidal energy. IET Renew. Power Gener., (December 2009):161-170, 2011.

[16] Aristeidis Zavvos, Alasdair Mcdonald, and Markus Mueller. Optimisation tools for large permanent magnet generators for direct drive wind turbines. IET Renew. Power Gener., (November 2012):163-171, 2013.

[17] R. S. Semken, M. Polikarpova, P. Roytta, J. Alexandrova, J. Pyrhonen, J. Nerg, A. Mikkola, and J. Backman. Direct-drive permanent magnet generators for high-power wind turbines: benefits and limiting factors. IET Renewable Power Generation, 6(1):1-8, January 2012.

[18] H.C. Lovatt. Analytical model of a classical switched-reluctance motor. IEE Proceedings Electric Power Applications, 152(2):352, 2005.

[19] J. Renedo Anglada and S. Sharkh. An insight into torque production and power factor in transverse-flux machines. IEEE Transactions on Industry Applications, PP(99):1-1, 2017.

[20] N. Boules. Prediction of no-load flux density distribution in permanent magnet machines. IEEE Transactions on Industry Applications, IA-21(3):633-643, May 1985.

[21] R. Rabinovici. Magnetic field analysis of permanent magnet motors. IEEE Transactions on Magnetics, (1):265-269, 1996.

[22] S. Taghipour Boroujeni, A. Abedini Mohammadi, A. Oraee, and H. Oraee. Approach for analytical modelling of axial-flux pm machines. IET Electric Power Applications, 10(6):441450, 2016.

[23] E. M. Freeman. The calculation of harmonics, due to slotting, in the flux-density waveform of a dynamo-electric machine. IEE Monograph, 109(523), 1962.

[24] D. Zarko, D. Ban, and T. A. Lipo. Analytical calculation of magnetic field distribution in the slotted air gap of a surface permanent-magnet motor using complex relative air-gap permeance. IEEE Transactions on Magnetics, 42(7):1828-1837, July 2006.

[25] K. Boughrara, B.L. Chikouche, R. Ibtiouen, D. Zarko, and O. Touhami. Analytical Model of Slotted Air-Gap Surface Mounted Permanent-Magnet Synchronous Motor With Magnet Bars Magnetized in the Shifting Direction. IEEE Transactions on Magnetics, 45(2):747-758, February 2009.

[26] K. Boughrara, D. Zarko, R. Ibtiouen, O. Touhami, and A. Rezzoug. Magnetic Field Analysis of Inset and Surface-Mounted Permanent-Magnet Synchronous Motors Using Schwarz Christoffel Transformation. IEEE Transactions on Magnetics, 45(8):3166-3178, 2009.

[27] A. Hanic, D. Zarko, and Z. Hanic. A novel method for no-load magnetic field analysis of saturated surface permanent-magnet machines using conformal mapping and magnetic equivalent circuits. IEEE Transactions on Energy Conversion, PP(99):1-10, 2016.

[28] J.R. Anglada and S.M. Sharkh. Analytical calculation of air-gap magnetic field distribution in transverse-flux machines. In Industrial Electronics (ISIE), 2016 International Symposium on, pages -, June 2016.

[29] M. Markovic, M. Jufer, and Y. Perriard. An exact formula for "tooth-to-tooth" permeance. IEEE Transactions on Magnetics, 41(7):2295-2299, July 2005. 